\title{
What we Can Learn from Neuroligin Gene Mutations and Autism Spectrum Disorders?
}

\author{
Antonelli Roberta ${ }^{1}$ and Paola Zacchi ${ }^{2 *}$ \\ ${ }^{1}$ Group of Translational Research in Child and Adolescent Cancer, Vall d'Hebron Research Institute (VHIR)-Universitat Autònoma de Barcelona (UAB), Spain \\ ${ }^{2}$ Department of Life Sciences, University of Trieste, Italy
}

*Corresponding author: Paola Zacchi, Department of Life Sciences, University of

Trieste, Trieste, Italy

Keywords: Neuroligins; Autism spectrum disorder (ASD); Trans-synaptic signaling

\section{Opinion}

The development of the human brain is a complex process that relies on the spatially and timely coordinated actions of genetic and environmental factors as well as experiential influences. Any alterations occurring during such developmental period can lead to various neuro-developmental disorders, being attention deficit hyperactivity disorder (ADHD), intellectual disability (ID) and autism spectrum disorders (ASD) the most common neuro-developmental abnormalities observed in childhood [1,2]. In particular ASD, estimated to affect up to $\sim 1$ in 110 children and being 4 times more frequent in males than females [3], encompasses a group of heterogeneous neuro-developmental diseases characterized by a very early onset of dysfunction in socialization (interaction, communication), restricted pattern of interests and stereotyped and repetitive behaviors. In some cases ASD is also associated with intellectual disability and mental retardation of variable degree of severity as well as epilepsy, anxiety, motor deficits and sleep abnormalities [4]. Even though it is now clear that ASD derives from a complex concurrence of genetics, epigenetic and environment factors, its genetic etiology is clearly recognized [5]. Monozygous twins and affected family studies demonstrate that genetic heritability contributes as much as 80$90 \%$ to the occurrence of autistic disorders. Large-scale genetic studies identified almost over a thousand genes associated with ASD and since autism is a spectrum of disorders it is conceivable that, in most cases, different genes act in concert to determine each patient's specific clinical manifestations [6]. Interestingly, many of the risk-genes identified in non-syndromic ASD cases are involved in various aspects of synapse development (formation and elimination), function and plasticity [7]. Mutations in genes coding for synaptic cell adhesion molecules, scaffolding proteins, and signaling adaptors such as Neuroligins (NLGN); Neurexins (NRXN), synaptic Ras GTPase-activating protein 1 (SYNGAP1); Fragile X Mental Retardation Protein (FMRP), SH3 and multiple ankyrin repeat domains (SHANK 2/3) [8], have been identified in several patients with ASD, to emphasize the concept that synaptic dysfunction at multiple levels may represent one of the major determinant of such brain disorders [9].

The first genes of which single point mutations were identified in non-syndromic ADS encode for members of the NLGN family of synaptic cell adhesion proteins. In human NLGNs are encoded by five genes, the autosomal NLGN1 and NLGN2, the X-located NLGN3 and NLGN4 and the Y-linked NLGN4 [10]. They all share a simple domain structure composed of an extracellular cholinesteraselike domain connected to a short intracellular C-terminal tail by a single transmembrane domain [11]. Despite the high sequence conservation, the different NLGN isoforms differ notably in their subcellular distribution, being their cytoplasmic domains able to recruit synapse specific scaffolding molecules. NLGN1 is exclusively present at excitatory synapses, NLGN2 and NLGN4 at inhibitory synapses while NLGN3 is present at both inhibitory as well as excitatory synapses ([12] and references herein). Natively NLGNs exist as dimers and dimerization is a pre-requisite for their efficient 
delivery at post-synapses where they can bind to presynaptically localized NRXNs. The formation of these trans-synaptic heterotetrameric NLGN/NRXN complexes establishes a functional crosstalk between pre-and post-synaptic specializations which is critical to promote synapse formation, organization, and remodeling. Therefore any gene mutation able to disrupt such trans-synaptic signaling is considered a key pathogenic event, based on the socalled trans-synaptic interaction hypothesis of ASD [13].

In 2003 Jamain and coworkers reported the identification of a missense mutation in NLGN3 (R451C) and a truncation in NLGNGN4 (396X) in affected siblings from two Swedish families [14]. From in vitro studies NLGN3R451C variant emerged to be strongly impaired in its delivery at synapses, being misfolded and mostly retained in the endoplasmic reticulum [15]. Engineered NLGN3R451C knockin (ki) mice were generated and deeply characterized [16]. In the behavioral examination NLGN3R451C mice displayed impaired social interaction while spatial learning abilities were enhanced [17]. The number of excitatory and inhibitory synapses were normal but mutant mice showed an increased inhibitory synaptic transmission in the somatosensory cortex $[16,17]$. Since NLGN3 knock-out (ko) mice did not exhibited such changes, a gain-of function phenotype was hypothesized. Interestingly NLGN3R451C mutation in the hippocampus caused, instead, a large increase in AMPA receptor-mediated excitatory synaptic transmission, and a dramatic change in NMDA receptor-mediated responses, leading to a gain-of-function phenotype. Collectively these findings support a synaptic impairment as a cause of ASD, whose contribution is strictly dependent on the brain region and on the circuitry involved.

Subsequently more than 50 distinct mutations in the NLGN4 locus have been linked to autism, making loss-of-function mutations in NLGN4 one of the most frequent monogenic causes of nonsyndromic autism known to date. Despite a great deal of experimental efforts carried out to unravel NLGN4 synaptic function, the results obtained are still quite puzzling. NLGN4 is poorly conserved in rodents and in mouse it is expressed at low levels and restricted to inhibitory glycinergic synapses in brain stem, retina, and spinal cord (CNS) [18]. In human, NLGN4 it is preferentially localized and functionally operative at excitatory synapses [19] and it is primarily expressed in cerebral cortex and parts of the striatum, brain structures involved in regulating higher cognitive functions, such as language, social interaction, and emotions, which are all affected in ASD. Once ectopically expressed in mouse or rat hippocampal neurons NLGN4 was shown to elicit a quite complex and diverse phenotype at excitatory synapses, questioning the use of animal models to drove conclusions possibly relevant for humans. In one family of two affected brothers Zhang and colleagues [20] identified a NLGN4 missense mutation leading to a single amino-acid substitution of a highly conserved arginine residue of the extracellular domain to tryptophan (R87W). Functionally this mutation dramatically impacts on NLGN4 folding and glycosylation processing, totally abolishing NLGN4 exit from the ER and its delivery at synaptic sites. While retaining the ability to interact with recombinant Nrnx, NLGN4R87W substitution fully abrogates NLGN4 ability to induce synapse formation in transfected neurons and halts NLGN4-induced suppression of excitatory synaptic transmission. As for NLGN3R451C mutant, functional inactivation of NLGN4 further emphasize the causal role of NLGN mutation in these neurodevelopmental disorders. Finally, among all NLG mutation identified in ASD patients, only a single mutation resides within the cytoplasmic sequences of NLGN4, the R704C substitution [21]. The NLGN4 R704C mutation was shown to affect the number of excitatory synapses by a postsynaptic action. Neuron expressing R704C exhibited a significant enhancement of synaptic strength manifested by an increase in the amplitude and frequency of AMPAR-mediated mEPSCs and in the amplitude of AMPAR-mediated evoked EPSCs, possibly controlling AMPA receptor trafficking in and outside the synapse. Also, in this case NLGN mutation drives a gain-of-function effect via a still poorly understood postsynaptic mechanism.

\section{Conclusion}

Genetic mutations occurring in NLGN have established a clear causative connection with ASD. Even though extremely rare, these mutations have offered to scientists a functional framework to further dissect all disturbances affecting the synaptic pathways involved in synaptic maturation, connectivity and stabilization. Given the complexity of the molecular machinery assembled at synapses, the way each NLGN mutant alter the overall functional connectivity in brain is still far from being comprehensive addressed. It is still essential to clarify how the very same NLGN mutation differentially impacts on neuronal structure, function and networks, leading to quite diverse clinical manifestations in different affected children. We still need to establish how NLGNspecific phenotypes at the molecular, cellular and neural circuitry levels promote the core behavioral deficits found in ASDs. Further advancement in our knowledge represents the unique path to better unravel the pathophysiological mechanisms involved in ASD etiology and to develop new effective therapeutic interventions that, nowadays, are quite limited.

\section{Acknowledgement}

None.

\section{Conflict of Interest}

No conflict of interest.

\section{References}

1. Verma V, Paul A, Amrapali Vishwanath A, Vaidya B, Clement JP (2019) Understanding intellectual disability and autism spectrum disorders from common mouse models: synapses to behaviour. Open Biol 9(6): 180265.

2. American Psychiatric Association (2013) Diagnostic and Statistical Manual of Mental Disorders ( $5^{\text {th }}$ edn) Arlington.

3. Elsabbagh M, Divan G, Koh YJ, Kim YS, Kauchali S, et al. (2012) Global prevalence of autism and other pervasive developmental disorders. $\mathrm{Au}$ tism Res 5(3): 160-179. 
4. Kiran Prasad Maski KP, Jeste SS, Spence SJ (2011) Common neurological co-morbidities in autism spectrum disorders. Curr Opin Pediatr 23(6): 609-615.

5. Beaudet AL (2007) Autism: highly heritable but not inherited. Nat Med 13(5): 534-536.

6. Woodbury-Smith M, Scherer SW (2018) Progress in the genetics of autism spectrum disorder. Developmental Medicine and Child Neurology 60(5): 445-451.

7. Betancur C, Sakurai T, Buxbaum JD (2009) The emerging role of synaptic cell-adhesion pathways in the pathogenesis of autism spectrum disorders. Trends Neurosci 32(7): 402-412.

8. Chen J, Yu S,Fu Y, Li X (2014) Synaptic proteins and receptors defects in autism spectrum disorders. Front Cell Neurosci 8: 276.

9. Brose N, O'Connor V, Skehel P (2010) Synaptopathy: dysfunction of synaptic function? Biochem Soc Trans 38(2): 443-444.

10. Craig AM, Kang Y (2007) Neurexin-neuroligin signaling in synapse development. Curr Opin Neurobiol 17(1): 43-52.

11. Lisé MF, El Husseini A (2006) The neuroligin and neurexin families: from structure to function at the synapse. Cell Mol Life Sci 63: 1833-1849.

12. Bemben MA, Shipman SL, Nicoll RA, Roche KW (2015) The cellular and molecular landscape of neuroligins. Trends in Neurosciences 38(8): 496-505.

13. Südhof TC (2008) Neuroligins and Neurexins Link Synaptic Function to Cognitive Disease. Nature 455(7215): 903-911.
14. Jamain S, Quach H, Betancur C, Råstam M, Colineaux C, et al. (2003) Mutations of the X-linked genes encoding neuroligins NLGN3 and NLGN4 are associated with autism. Nat Genet 34(1): 27-29.

15. De Jaco A, Lin MZ, Dubi N, Comoletti D, Miller MT, et al. (2010) Neuroligin trafficking deficiencies arising from mutations in the alpha/beta-hydrolase fold protein family. J Biol Chem 285(37): 28674-28682.

16. Tabuchi K, Blundell J, Etherton MR, Hammer RE, Liu X, et al. (007) A neuroligin-3 mutation implicated in autism increases inhibitory synaptic transmission in mice. Science 318(5847): 71-76.

17. Chadman KK, Gong S, Scattoni ML, Boltuck SE, Gandhy SU, et al. (2008) Minimal aberrant behavioral phenotypes of neuroligin-3 R451C knockin mice. Autism Res 1(3): 147-158.

18. Hoon M, Soykan T, Falkenburger B, Hammer M, Patrizi A, et al. (2011) Neuroligin-4 is localized to glycinergic postsynapses and regulates inhibition in the retina. PNAS 108(7): 3053-3058.

19. Marro SG, Chanda S, Yang N, Janas JA, Valperga G, et al. (2019) Neuroligin-4 Regulates Excitatory Synaptic Transmission in Human Neurons. Neuron 103(4): 617-626.e6

20. Zhang C, Milunsky JM, Newton S, Ko J, Zhao G, et al. (2009) A neuroligin-4 missense mutation associated with autism impairs neuroligin-4 folding and endoplasmic reticulum export. J Neurosci 29(35): 10843-10854.

21. Yan J, Oliveira G, Coutinho A, Yang C, Feng J, et al. (2005) Analysis of the neuroligin 3 and 4 genes in autism and other neuropsychiatric patients. Mol Psychiatry 10(4): 329-332. 